\title{
A NOTE ON MEASURE FUNCTIONS IN A LATTICE ${ }^{1}$
}

\author{
M. F. SMILEY
}

We give first an equivalent statement of the measurability criterion of Carathéodory ${ }^{2}$ which is applicable to an arbitrary lattice. We then study the closure with respect to finite and denumerable sums and products of the subset of measurable elements of a modular lattice. The case of regular ${ }^{3}$ "outer measure functions" is then briefly discussed. The elements of the theory of lattices are presupposed. ${ }^{4}$

Let us consider a lattice $L$ on which is defined a real-valued function $\mu(a)$. The elements $a \varepsilon L$ which satisfy

$$
\mu(a+b)+\mu(a b)=\mu(a)+\mu(b)
$$

for every $b \varepsilon L$ will be called $\mu$-measurable. The totality of $\mu$-measurable elements will be denoted by $L(\mu)$.

REMARK 1. If $L$ is a Boolean algebra and $\mu(0)=0$, then $a \varepsilon L(\mu)$ if and only if $a \varepsilon L$ and satisfies the condition of Caratheodory, ${ }^{5}$ that is,

$$
\mu(b)=\mu(a b)+\mu(b-a b)
$$

for every $b \varepsilon L$. For, if $a \varepsilon L$ satisfies (1), the equation (1) and

$$
\mu(a+(b-a b))+\mu(0)=\mu(a)+\mu(b-a b)
$$

yield (2). The converse is proved by Caratheodory. ${ }^{\circ}$

THeORem 1. If $L$ is a modular lattice, then $L(\mu)$ is a sublattice of $L$.

Proof. Let $a, c \varepsilon L(\mu), b \varepsilon L$. We obtain successively

$$
\begin{aligned}
\mu(a+(c+b))+\mu(a(c+b)) & =\mu(a)+\mu(c+b) \\
& =\mu(a)+\mu(c)+\mu(b)-\mu(c b) \\
& =\mu(a+c)+\mu(b)+\mu(a c)-\mu(c b) .
\end{aligned}
$$

Since $c \varepsilon L(\mu)$ we have

1 Presented to the Society, September 5, 1939.

2 Vorlesungen uber Reelle Funktionen, 2d edition, p. 246.

3 Ibid., p. 258.

${ }^{4}$ See, for example, G. Birkhoff, On the combination of subalgebras, Proceedings of the Cambridge Philosophical Society, vol. 29 (1933), pp. 441-464; O. Ore, On the foundations of abstract algebra I, Annals of Mathematics, (2), vol. 36 (1935), pp. 406437. The terminology and notation are those used by L. R. Wilcox and the author, Metric lattices, Annals of Mathematics, (2), vol. 40 (1939), pp. 309-327.

5 Op. cit., p. 246.

6 Ibid., p. 252. 


$$
\begin{aligned}
& \mu(c+a(c+b))+\mu(a c)=\mu(c)+\mu(a(c+b)), \\
& \mu(c+(a+c) b)+\mu(c b)=\mu(c)+\mu((a+c) b) .
\end{aligned}
$$

Using the modular law we see that $(a+c)(c+b)=c+a(c+b)=c$ $+(a+c) b$. It is then clear that

$$
\mu(a c)-\mu(c b)=\mu(a(c+b))-\mu((a+c) b),
$$

and (1) with $a$ replaced by $a+c$ follows easily. Thus $a+c \varepsilon L(\mu)$. By duality, $a c \varepsilon L(\mu)$. This completes the proof.

Definition 1. If, for each increasing (decreasing) sequence $\left(a_{i}\right.$; $i=1,2, \cdots)$ of elements of $L(\mu)$ with a sum (product) a $\varepsilon L$, we have $\lim \mu\left(a_{i}\right)=\mu(a)$ as $i \rightarrow \infty$ we say that ${ }^{7} L$ satisfies $B^{+}(\mu)\left(B^{-}(\mu)\right)$; if moreover $\lim \mu\left(a_{i}+b\right)=\mu(a+b)$ and $\lim \mu\left(a_{i} b\right)=\mu(a b)$ as $i \rightarrow \infty$ for each $b \varepsilon L$, we say that $L$ satisfies $B^{+}\left(B^{-}\right)$.

REMARK 2. If $L$ satisfies $B^{+}\left(B^{-}\right)$, then $L$ satisfies $B^{+}(\mu)\left(B^{-}(\mu)\right)$. It suffices to take $b=a$ in the definition of $B^{+}\left(B^{-}\right)$.

We shall assume throughout the remainder of this note that $L$ is modular and that $\mu(a)$ is monotone increasing.

THEOREM 2. A sufficient condition for closure of $L(\mu)$ with respect to denumerable sums (products) in $L$ is that $L$ satisfy $B^{+}\left(B^{-}\right)$. This condition is necessary if $L$ satisfies $B^{+}(\mu)\left(B^{-}(\mu)\right)$.

Proof. To show that $B^{+}$is sufficient, consider a sequence $\left(a_{i}\right.$; $i=1,2, \cdots)$ of elements of $L(\mu)$ with a sum $a \varepsilon L$. Define $c_{i} \equiv \sum\left(a_{j}\right.$; $j=1,2, \cdots, i)$. Clearly $a=\sum c_{i}$, and $\left(c_{i} ; i=1,2, \cdots\right)$ is increasing. By Theorem 1, $c_{i} \varepsilon L(\mu)$ for each $i=1,2, \cdots$, and hence $\mu\left(c_{i}+b\right)$ $+\mu\left(c_{i} b\right)=\mu\left(c_{i}\right)+\mu(b)$ for each $b \varepsilon L$. On taking the limit and using $B^{+}$ we see that $\mu(a+b)+\mu(a b)=\mu(a)+\mu(b)$. Thus $a \varepsilon L(\mu)$, and $B^{+}$is sufficient. For the necessity, consider an increasing sequence $\left(a_{i}\right.$; $i=1,2, \cdots)$ ) of elements of $L(\mu)$ with a sum $a \varepsilon L$. For each $b \varepsilon L$ and each $i=1,2, \cdots, a+b \geqq a_{i}+b$ and $a b \geqq a_{i} b$; and hence $\mu(a+b)$ $\geqq \mu\left(a_{i}+b\right), \mu(a b) \geqq \mu\left(a_{i} b\right)$. Define $\alpha \equiv \lim \mu\left(a_{i}+b\right)$ and $\beta \equiv \lim \mu\left(a_{i} b\right)$ as $i \rightarrow \infty$. Since $a_{i} \varepsilon L(\mu)$ we have $\mu\left(a_{i}+b\right)+\mu\left(a_{i} b\right)=\mu\left(a_{i}\right)+\mu(b)$. On taking the limit and using $B^{+}(\mu)$ and the fact that $a \varepsilon L(\mu)$ we obtain $\alpha+\beta=\mu(a)+\mu(b)$. It follows that $\alpha=\mu(a+b), \beta=\mu(a b)$. Thus $B^{+}$ is necessary when $L$ satisfies $B^{+}(\mu)$. The alternate reading is dual. The proof is complete.

Definition 2. (1) For each $a \varepsilon L$ we define $\mu^{+}(a) \equiv$ g.1.b. $[\mu(c)$; $c \varepsilon L(\mu), c \geqq a], \mu^{-}(a) \equiv$ l.u.b. $[\mu(c) ; c \varepsilon L(\mu), c \leqq a]$.

${ }^{7}$ Cf. L. R. Wilcox and the author, op. cit., p. 317. 
(2) We say that $\mu(a)$ is outer (inner) regular in case $\mu(a)=\mu^{+}(a)$ $\left(\mu(a)=\mu^{-}(a)\right)$ for every $a \varepsilon L$.

LEMMA 1. If $\mu(a)$ is outer regular, then

$$
\mu(a+b)+\mu(a b) \leqq \mu(a)+\mu(b)
$$

for every $a, b \varepsilon L$.

Proof. Consider $a, b \varepsilon L$. For each $c, d \varepsilon L(\mu)$ for which $c \geqq a, d \geqq b$ we have $c+d \geqq a+b, c d \geqq a b$; and, by Theorem $1, c+d, c d \varepsilon L(\mu)$. Consequently, since $\mu(a)$ is outer regular, $\mu(a+b)+\mu(a b) \leqq \mu(c+d)$ $+\mu(c d)=\mu(c)+\mu(d)$. The lemma follows by applying a simple property of the greatest lower bound.

Theorem 3. If $L$ satisfies $B^{+}(\mu)\left(B^{-}(\mu)\right)$ and $\mu(a)$ is outer (inner) regular, then $L$ satisfies $B^{+}\left(B^{-}\right)$.

Proof. This follows from Lemma 1 and its dual by the method used in proving Theorem 2.

We now assume that $L$ is closed with respect to denumerable sums and products.

LEMMA 2. If $L$ satisfies $B^{-}\left(B^{+}\right)$, then for each a $\varepsilon L$ there is an element $c \varepsilon L(\mu)$ such that $c \geqq a(c \leqq a)$ and $\mu(c)=\mu^{+}(a)\left(\mu(c)=\mu^{-}(a)\right)$.

Proof. This is an easy consequence of Theorem 2.

REMARK 3. It is now clear that when $L$ satisfies $B^{-}$and $\mu(a)$ is outer regular the distance function ${ }^{9} \delta(a, b)=2 \mu(a+b)-\mu(a)-\mu(b)$ identifies each $a \varepsilon L$ with an element $c \varepsilon L(\mu)$.

THEOREM 4. If $L$ satisfies $B^{+}\left(B^{-}\right)$and $\mu(a)$ is outer (inner) regular, then an element $a \varepsilon L$ belongs to $L(\mu)$ if and only if $\mu^{-}(a)=\mu(a)$ $\left(\mu^{+}(a)=\mu(a)\right)$.

Proof. Consider an element $a \varepsilon L$ for which $\mu^{-}(a)=\mu(a)$. By hypothesis and Lemma 2 there is an element $c \varepsilon L(\mu)$ such that $c \leqq a$ and $\mu(c)=\mu^{-}(a)$. Thus, for each $b \varepsilon L, \mu(a)+\mu(b)=\mu^{-}(a)+\mu(b)=\mu(c)$ $+\mu(b)=\mu(c+b)+\mu(c b) \leqq \mu(a+b)+\mu(a b)$. Consequently, by Lemma 1, $a \varepsilon L(\mu)$. The converse is trivial. The alternate reading is dual. The proof is complete.

LEHIGH UNIVERSITY

${ }^{8}$ Cf. Carathéodory, op. cit., p. 258.

- See L. R. Wilcox and the author, op. cit., p. 311. 